\title{
Neuroinflammation and brain functional disconnection in Alzheimer's disease
}

\author{
Francesca Baglio ${ }^{1 *}{ }^{*}$, Marina Saresella $^{1 \dagger}$, Maria Giulia Preti ${ }^{1,2}$, Monia Cabinio $^{1,3}$, Ludovica Griffanti ${ }^{1,2}$, \\ Ivana Marventano ${ }^{1}$, Federica Piancone ${ }^{1}$, Elena Calabrese ${ }^{1}$, Raffaello Nemni ${ }^{1,3}$ and Mario Clerici ${ }^{1,3}$ \\ I IRCCS, Don Gnocchi Foundation, Milan, Italy \\ 2 Department of Electronics, Information and Bioengineering, Politecnico di Milano, Milan, Italy \\ ${ }^{3}$ Università degli Studi di Milano, Milan, Italy
}

\section{Edited by:}

Antonio Camins, University of

Barcelona, Spain

\section{Reviewed by:}

Lourdes Lorigados, International

Center for Neurological Restoration,

Cuba

Sandra Orozco-Suárez, Mexican

Institute of Social Security, Mexico

*Correspondence:

Francesca Baglio, Magnetic

Resonance Laboratory and

Neurorehabilitation Unit, Fondazione

Don Carlo Gnocchi ONLUS, IRCCS S.

Maria Nascente, Via Capecelatro 66,

20148 Milan, Italy

e-mail: fbaglio@dongnocchi.it

${ }^{\dagger}$ Francesca Baglio and Marina

Saresella have contributed equally to

this work.
Neuroinflammation and brain functional disconnection result from $\beta$-amyloid (A $\beta$ ) accumulation and play fundamental roles in the pathogenesis of Alzheimer's disease (AD). We investigated possible correlations between these two AD-associated phenomena using DTI-based tractography and immunologic analyses in people with amnestic mild cognitive impairment (aMCI) and AD. DTI-Analyses focused on corpus callosum (CC). We found that frontal $\mathrm{CC}$ regions were preserved with respect to the posterior ones in $\mathrm{aMCl}$; in these individuals significant correlations were seen between DTI-derived metrics in frontal-parietal $C C$ areas and $A \beta_{42}$-stimulated BDNF-producing CD4+T lymphocytes and PDL-1-expressing CD14+ cells. These associations were lost in AD where DTI data involving the same CC areas correlated instead with $A \beta_{42}$-stimulated interleukin (IL)-21 producing CD4+ T lymphocytes. Higher susceptibility to PDL-1-mediated apoptosis of $A \beta_{42}$-specific lymphocytes and BDNF-associated survival of existing neurons could contribute to the relative CC structure preservation seen in $\mathrm{aMCl}$. These potentially protective mechanisms are lost in frank $A D$, when severe alterations in the $C C$ are mirrored in peripheral blood by proinflammatory cytokines-producing T cells. Monitoring of immune cells in peripheral blood could have a prognostic value in AD.

Keywords: Alzheimer's disease, mild cognitive impairment, magnetic resonance imaging, diffusion tensor imaging, immunology, neuroinflammation

\section{INTRODUCTION}

Alzheimer's disease $(\mathrm{AD})$ is a neurodegenerative disorder involving both gray matter (GM) and white matter (WM) tissues that is now well defined as a part of a continuum of clinical and biological phenomena. $\mathrm{AD}$ is characterized by the accumulation of amyloidbeta $(A B)$ peptide into amyloid plaques in the extracellular brain parenchyma and by the formation of neurofibrillary tangles (NFT) within neurons as a result of the abnormal phosphorylation of the microtubules-associated tau-protein. Neuroinflammation is strongly suspected to play an important role in $\mathrm{AD}$ that might be the culprit of the disease or, possibly, a reaction to the pathology (Ke et al., 2006; Zipp and Aktas, 2006). Thus, both increased concentrations of proinflammatory cytokines (Bauer et al., 1991; Strauss et al., 1992; Remarque et al., 2001; Bermejo et al., 2008) and changes in lymphocyte subsets, with an augmented percentage of activated immune cells are described in $\mathrm{AD}$ (Lombardi et al., 1999; Speciale et al., 2007; Saresella et al., 2011). In particular, recent results indicated that a significant reduction of PD-1- and PDL1 -expressing cells is present in $\mathrm{AD}$ and amnestic mild cognitive impairment (aMCI), a prodromal stage of $\mathrm{AD}$. The interaction between these molecules is responsible for the induction of tolerance and for the apoptosis of antigen-specific cells (Francisco et al., 2010); as the impairment seen in $\mathrm{AD}$ and $\mathrm{aMCI}$ is specific for $A \beta$-stimulated cells, this alteration could play a role in the neuroinflammation accompanying AD (Saresella et al., 2012).
Functional disconnection as a consequence of $A \beta$ pathology has also recently been hypothesized to be a major mechanism in AD clinical evolution (Drzezga et al., 2011). This suggestion stems from the evidence that WM damage can be observed even in $\mathrm{AD}$ patients in early stages of disease, and this correlates with clinical measures of cognitive disability (Parente et al., 2008; Bozzali et al., 2011). DTI-based tractography is an ideal MRI based technique for the study of WM changes in the most relevant fiber bundles of the brain (Le Bihan, 2003), and DTI results of previous studies confirmed that brain disconnection plays an important role in $\mathrm{AD}$ pathophysiology, and contributes to the progressive accumulation of disabilities in the transitional stage between normal aging and dementia (Bozzali et al., 2002, 2011; Takahashi et al., 2002; Duan et al., 2006; Naggara et al., 2006; Zhang et al., 2007; Serra et al., 2010; Gili et al., 2011).

The corpus callosum (CC), the largest WM fiber connection of the human brain, is an ideal target to test physiopathological changes in AD. In a previous DTI-study (Preti et al., 2012), we investigated the pattern of CC abnormalities in different stages of $\mathrm{AD}$ and showed the presence of an early selective damage of the central and posterior CC subregions in this disease. This CC damage pattern is consistent both with the distribution of GM loss found in volumetric studies (Karas et al., 2008; Kakeda and Korogi, 2010; Vemuri et al., 2011), and with the neuropsychological profile of the patients (Leube et al., 2008; Risacher et al., 2009; Serra et al., 
2010). These alteration are also consistent with the pathological knowledge that we have of AD progression, according to which the posterior CC subregions are preferentially involved in the earlier stages of $\mathrm{AD}$ whereas and the anterior $\mathrm{CC}$ subregions are usually affected in the later stages of disease (Brun and Englund, 2002).

We investigated possible correlations between neuroinflammatory parameters and brain disconnection in $\mathrm{AD}$ and aMCI using DTI-based tractography and immunologic analyses performed on peripheral blood mononuclear cells. In particular, the CC was evaluated in its different parts to identify the anatomical regions in which the damage mediated by inflammation plays a prominent role, and immunologic studies were performed in peripheral blood in the attempt to identify easily reproducible diagnostic and/or prognostic parameters.

\section{MATERIALS AND METHODS SUBJECTS}

Forty patients diagnosed with probable $\mathrm{AD}$ according to the NINCDS-ADRDA criteria (McKhann et al., 2011) and 20 subjects diagnosed with aMCI according to Petersen criteria (Petersen, 2004) were included in the study. All subjects were consecutively recruited at the Fondazione Don Gnocchi, IRCCS in Milano, Italy. $\mathrm{AD}$ patients were in mild stage of the disease as determined by both clinical dementia rating (CDR) (Morris, 1993) scale (CDR range 0.5-1.5) and mini-mental state examination score (Magni et al., 1996) (MMSE mean/SD 20.2/2.7). To be eligible for the study, aMCI individuals were required to meet the Grundman operational criteria (Grundman et al., 2004): memory complaint, confirmed by an informant; abnormal memory function, documented by previous extensive neuropsychological evaluation; normal general cognitive function, as determined by both CDR scale (Morris, 1993) (CDR with at least a 0.5 in the memory domain) and MMSE (Magni et al., 1996) score (MMSE greater than or equal to 24); no impairment in functional activities of daily living as determined by a clinical interview with the patient and informant; no significant cerebral vascular disease (Hachinski score less than or equal to 4) (Rosen et al., 1980); no major psychiatric illnesses with particular attention to exclude subjects with history of depression (Hamilton Depression Rating Scale score less than or equal to 12) (Hamilton, 1960). To increase the diagnostic accuracy analyses of hippocampal volumes, an index of downstream neural injury according to the guidelines for MCI due to Alzheimer's dementia (Sperling et al., 2011), were also included in the study. Demographic details of the study sample are summarized in Table 1. Twenty-five healthy controls (CTRs) that were age-and sex-matched to the $\mathrm{AD}$ patients $(70.2 \pm 5.1$ years; $11 \mathrm{M} / 14 \mathrm{~F}$; MMSE score $>26$ ) were also included in the MR analysis for the tractographic atlas construction. The study conformed to the ethical principles of the Helsinki Declaration; all patients or their care-givers gave informed consent according to a protocol approved by the local ethics committee of the Don Gnocchi Foundation.

\section{MRI ACQUISITIONS AND ANALYSES MRI acquisition protocol}

Brain MR images were acquired using a 1.5- $\mathrm{T}$ scanner (Siemens Magnetom Avanto, Erlangen, Germany). The following
Table 1 | Demographical and anatomical information of the sample

\begin{tabular}{|c|c|c|c|}
\hline & AD & aMCI & CTR \\
\hline N & 40 & 20 & 25 \\
\hline Age [years, mean (SD)] & $73.7(12)$ & $73.9(5.4)$ & $70.2(5.1)$ \\
\hline Sex (M:F) & $17: 23$ & $12: 8$ & $11: 14$ \\
\hline MMSE score [mean (SD)] & $20.2(2.7)(\S)$ & $25.6(1.7)\left(^{\wedge}\right)$ & 29.1 (0.7) (\#) \\
\hline CDR (range) & $1-1.5(\S)$ & $0-0.5$ & 0 \\
\hline $\begin{array}{l}\text { Right hippocampal } \\
\text { volume }\left[\mathrm{mm}^{3} \text {, mean (SD)] }\right.\end{array}$ & 2898 (575) (§) & $3158(519)(\wedge)$ & 3774 (558) (\#) \\
\hline $\begin{array}{l}\text { Left hippocampal volume } \\
{\left[\mathrm{mm}^{3} \text {, mean (SD)] }\right.}\end{array}$ & $2719(482)(\S)$ & $3065(489)(\wedge)$ & 3607 (450) (\#) \\
\hline
\end{tabular}

$A D$, Alzheimer's disease; aMCl, amnestic mild cognitive impairment; CTR, healthy controls; MMSE, mini-mental state evaluation; CDR, clinical dementia rating scale; SD, standard deviation. Chi-squared test was used for gender comparison and one-way ANOVA with Bonferroni post hoc test was used for age, MMSE score and hippocampal volumes comparisons (significance level: $p<0.05)$. (\#) Significant compared to $\mathrm{MCl}$ and $\mathrm{AD}$ group; (§) significant compared to $\mathrm{MCl}$ and CTR; $(\wedge)$ significant compared to $A D$ and CTR.

sequences were acquired: (1) dual-echo turbo spin echo $(\mathrm{TR} / \mathrm{TE}=2920 / 22 \mathrm{~ms}, \mathrm{FoV}=240 \mathrm{~mm} \times 180 \mathrm{~mm}$, in-plane resolution $=0.75 \mathrm{~mm} \times 0.75 \mathrm{~mm}$, slice thickness $=4 \mathrm{~mm}$, number of axial slices $=25)$ and FLAIR sequence $(\mathrm{TR} / \mathrm{TE}=9000 / 121 \mathrm{~ms}$, FoV $=240 \times 168 \mathrm{~mm}$, in-plane resolution $=0.94 \mathrm{~mm} \times 0.94 \mathrm{~mm}$, slice thickness $=5 \mathrm{~mm}$, number of coronal slices $=24$ ), to exclude patients showing WM hyperintensities outside the normal range; (2) three-dimensional T1-weighted magnetization prepared rapid gradient echo (MPRAGE) $(\mathrm{TR} / \mathrm{TE}=1900 / 3.37 \mathrm{~ms}$, FoV $=192 \mathrm{~mm} \times 256 \mathrm{~mm}$, in-plane resolution $1 \mathrm{~mm} \times 1 \mathrm{~mm}$, slice thickness $=1 \mathrm{~mm}$, number of axial slices $=176)$; (3) diffusion-weighted pulsed-gradient spin-echo planar $(\mathrm{TR} / \mathrm{TE}=$ $7000 / 94 \mathrm{~ms}, \quad F O V=320 \mathrm{~mm} \times 240 \mathrm{~mm}$, in-plane resolution $=$ $2.5 \mathrm{~mm} \times 2.5 \mathrm{~mm}$, slice thickness $=2.5 \mathrm{~mm}$, number of axial slices $=50)$, with diffusion gradients $\left(b\right.$-value $\left.=900 \mathrm{~s} / \mathrm{mm}^{2}\right)$ applied in 12 non-collinear directions. Two runs of DW images with one $b=0$ image without diffusion weighting were acquired for each subject.

\section{Analysis of T1-weighted structural images}

Two different analyses were performed on the T1-weighted structural images in order to provide further information about the underline pathology in this cohort of subjects: (1) computation of hippocampal volumes; (2) voxel-based morphometry (VBM) analysis.

Hippocampal volume data were extracted for each subject from high-resolution T1 3D images. Segmentation of right and left hippocampi was performed using FSL's FIRST method (Patenaude et al., 2011), an approach combining both shape and intensity information within a Bayesian Model to segment subcortical structures. After hippocampal segmentation, volumetric data had been obtained in each subject using a specific FSL function. 
The VBM analysis was conducted using VBM $8^{1}$, toolbox of SPM8 ${ }^{2}$, running on Matlab 7.6.0 $0^{3}$. VBM was conducted according to the Unified Method (Ashburner and Friston, 2005). After GM segmentation all images underwent spatial smoothing using a Gaussian kernel (FWHM $8 \mathrm{~mm}$ ).

\section{Analysis of diffusion-weighted images}

Diffusion-Weighted Images (DWI) were first corrected for eddy current distortions, using FSL ${ }^{4}$. For every subject, the images of the second run were registered to the first one using SPM5 (see text footnote 2). The affine transformation between the $b=0$ image of the second run and the $b=0$ image of the first one was estimated and applied to all the DWI of the second run. From the DWI, the diffusion tensor (DT) and its scalar invariants fractional anisotropy (FA) and mean diffusivity (MD) were computed for every voxel, using Diffusion Toolkit ${ }^{5}$ v0.6, which firstly rotates the B-matrix for slice angulation and for the rotation applied by FSL and SPM.

\section{CC tractographic analysis}

To assess CC integrity, the atlas-based tractography method described in Preti et al. (2012) was adopted. For the atlas construction, individual deterministic tractography of the CC was performed for the CTRs with Diffusion Toolkit, using all voxels as seed points (brute force approach) and the Interpolated Streamline algorithm. FA and angle thresholds of 0.2 and $35^{\circ}$ respectively were adopted as stopping criteria for the tractographic algorithm. The tracts of seven $\mathrm{CC}$ regions portions (CC1: orbital frontal, CC2: anterior frontal, CC3: superior frontal, CC4: superior parietal, CC5: posterior parietal, CC6: temporal, CC7: occipital) were selected from the whole brain tracts by drawing regions of interest (ROIs) on the FA map using Trackvis v0.5.1 (see text footnote 6) and following the ROI positioning suggested by Lebel et al. (2010). From the individual tractographic reconstructions, the probabilistic atlases of the seven CC portions were obtained, adopting the method described in detail in Preti et al. (2012).

Average FA and MD values along the tracts in the seven CC portions (CC1-CC7) were extracted for every subject basing on the locations given by the created tractographic atlases. First, all subjects were linearly registered to the atlas space with FSL, using the FMRIB58_FA as template for the alignment. Then, the estimated transformation between individual FA maps and the template was applied to both FA and MD maps of every subject. Finally, the registered FA and $\mathrm{MD}$ maps of all subjects were masked with the atlases and, for each tract portion, the index mean values weighted for the probability given by the atlas were computed.

\section{IMMUNOLOGICAL ANALYSES}

\section{Blood sample collection and cell culture}

Whole blood was collected in vacutainer tubes containing ethylenediaminetetraacetic acid (EDTA) (Becton Dickinson \& Co.,

\footnotetext{
${ }^{1}$ http://dbm.neuro.uni-jena.de/vbm/

${ }^{2}$ http://www.fil.ion.ucl.ac.uk/spm/

${ }^{3}$ http://www.mathworks.it/products/matlab

${ }^{4}$ http://www.fmrib.ox.ac.uk/fsl/

${ }^{5}$ www.trackvis.org
}

Rutherford, NJ, USA). PBMC were separated on lympholyte separation medium (Cedarlane, Hornby, ON, Canada); viable leukocytes were determined using a Scepter 2.0 Handheld Automated Cell Counter (Millipore, Billerica, MA, USA).

About $1 \times 106$ PBMC were cultured for $24 \mathrm{~h}$ in RPMI $1640+10 \%$ human serum, $2 \mathrm{mM}$ L-glutamine, and $1 \%$ penicillin (Invitrogen Ltd, Paisley, UK) with either non-immunogenic peptides (Saresella et al., 2008) or with $10 \mu \mathrm{g} / \mathrm{ml}$ of $\mathrm{A} \beta_{42}$-peptide soluble monomer (Sigma, St Louis, MO, USA) at $37^{\circ} \mathrm{C}$ in a humidified 5\% CO2 atmosphere for $24 \mathrm{~h}$. The dose of peptides was chosen based on previously results (Saresella et al., 2010). For cytokine analyses, $10 \mu \mathrm{g} / \mathrm{ml}$ of brefeldin A (Sigma-Aldrich) was added to the cell cultures during the last $6 \mathrm{~h}$ of stimulation to block protein secretion.

\section{Flow-cytometry immunofluorescent staining}

$\mathrm{A} \beta_{42}$-peptide - or non-immunogenic peptides-stimulated PBMC were resuspended in PBS and stained with anti-CD4, -CD8, CD19, -CD14, -PD-1, or -PDL-1 specific mAbs (eBioscience, San Diego, CA, USA). Cells were washed after $30 \mathrm{~min}$ incubation at room temperature in the dark, treated with FIX and PERM (FIX and PERM Cell Permeabilization kits; eBioscience) and incubated for $30 \mathrm{~min}$ at $4^{\circ} \mathrm{C}$ in the dark with cytokine-specific mAbs.

The following mAbs were used: phycoerythrin (PE)-Cyanin-7 (PC7)-labeled-anti-CD4 (clone SFCI12T4D11; mouseIgG1), PECyanin-5 (PC5)-labeled-anti-CD8 (clone SFC12Thy2D3; mouse$\mathrm{IgG}_{1}$ ), PC5-labeled-anti-CD19 (clone J4.119; mouse $\mathrm{IgG}_{1}$ ), and PC7-labeled-anti CD14 (clone RMO52, mouse $\mathrm{IgG}_{2 \mathrm{a}}$, Beckman-Coulter Brea, CA, USA); PE-labeled-anti-PD-1 (clone MIH4; mouse-IgG 1 ) and (PC5)-labeled-anti-PDL-1 (clone MIH1; mouse-IgG1; eBioscience Cornerstone Court, West, San Diego, CA, USA). The intracellular molecule detection mAbs used were: PE-labeled-anti-interleukin (IL)-6 (clone 1936, mouse IgG $_{2 B}$ ), PE-labeled-anti-IL-10 (clone JES9D7; mouse IgG 1 ), PE-labeledanti-IL-22 (clone 142928, mouse IgG1), Fluorescein isothiocyanate (FITC)-labeled-anti-IL-12 (clone 27537, mouse $\operatorname{IgG}_{1}$ ), PE-labeled-anti-TGF- $\beta$ - (clone 9016, mouse IgG1), PE-labeledanti-IFN $\gamma$ (clone 25723, mouse $\operatorname{IgG}_{2 b}$ ), and PE-labeled-antiBDNF (clone 35909; mouse-IgG1 R\&D Systems, Inc., Minneapolis, MN, USA); PE-labeled-anti-IL-9 (clone MH9A4, mouse IgG2B,k), PE- labeled-anti-IL-23 (clone C11.5, mouse $\operatorname{IgG}_{1 \mathrm{k}}$ ), and PC5-labeled-anti-IL-17 (clone BL168, mouse IgG1k; Biolegend San Diego, CA, USA); PE-labeled-anti-ROR-C/ $\gamma$ (clone AFKJS-9, rat IgG2a), PE-labeled- anti T-bet- (clone 39D, mouse IgG1), PE-labeled-anti-GATA-3 (cloneTWAY, rat IgG2B,k), and PE-labeled-anti-IL-21 (clone 3A3-N2, mouse IgG1; Bioscience).

\section{Flow-cytometry analysis}

A Beckman-Coulter Cytomics FC-500 flow cytometer equipped with a single $15 \mathrm{~mW}$ argon ion laser operating at $488 \mathrm{~nm}$ and interfaced with CXP Software 2.1 was used. Two-hundred-thousand events were acquired and gated on lymphocyte or monocyte FSC and SSC properties. Samples were first run using isotype control or single fluorochrome-stained preparations for color compensation. Rainbow Calibration Particles (Spherotec, Inc. Lake Forest, IL, USA) were used to standardize flow-cytometry results. 


\section{STATISTICAL ANALYSIS}

The statistical analyses were accomplished using commercial software (SPSS for Windows, V 18.0; SPSS Inc). We compared aMCI, $\mathrm{AD}$, and CTRs on demographic data, using the chi-square test and one-way ANOVA with Bonferroni post hoc test for categorical and dimensional variables, respectively. To test the comparisons between the aMCI and AD groups, Mann-Whitney $U$ - or $t$-test were performed and continuous variables were described using median and interquartile range, according to variable skewness. The statistical correlations between immunological parameters and RM data were investigated by means of Spearman correlation coefficient and 95\% confidence limits performed by Fisher's $Z$ transformation.

\section{RESULTS \\ DEMOGRAPHICAL AND ANATOMICAL CHARACTERISTICS OF THE PARTICIPANTS}

CTRs, aMCI subjects, and AD patients were comparable for age and gender; a significant difference was found instead for MMSE and CDR values, accordingly with the adopted inclusion criteria (Table 1).

Structural MRI confirmed the pattern of GM atrophy typical of aMCI and AD. VBM showed that patients with $\mathrm{AD}$ were significantly more atrophic than aMCI in several brain regions including medial, anterior, and postero-inferior regions of temporal lobes (left $>$ right) and precuneus/posterior cingulate (Figure 1). Hippocampal volumes of CTRs were significantly different compared to aMCI and $\mathrm{AD}(p<0.05$ Bonferroni post hoc; CTRs $>$ aMCI $>$ AD) .

\section{DTI ANALYSES}

Significantly reduced FA values were found in the frontal CC regions (CC2-CC3) of AD compared to aMCI subjects $(p<0.01)$. Moreover, $\mathrm{MD}$ values were significantly higher in $\mathrm{AD}$ with respect to aMCI group, in every portion with the exception of CC7. Results of the comparison between mean FA/MD computed in the seven $\mathrm{CC}$ portions of the two groups (aMCI and AD patients) are showed in Table 2 and Figure 2.

\section{IMMUNOLOGICAL RESULTS}

Cytokine-producing and transcription factor-expressing T cells in $A \beta_{42}$-peptide-stimulated cell cultures

PBMC obtained from $\mathrm{AD}$ and aMCI patients were stimulated in vitro with either $A \beta_{42}$-peptide or with non-immunogenic control peptides. Cytofluorimetric analyses were used to analyze IFN $\gamma$-, IL-9-, IL-17-, IL-21-, IL-22-, and BDNF-producing CD4+ and CD8+ T lymphocytes. Results showed that, whereas no differences were seen when cells were stimulated with non-immunogenic peptides (data not shown), $\mathrm{A} \beta_{42}$-stimulatedBDNF-producing CD4+ T lymphocytes were significantly reduced in $\mathrm{AD}$ compared to aMCI $(p=0.003)$; interestingly, and confirming the increased inflammatory status of AD, IFN $\gamma$ producing-CD8 + T lymphocytes were increased in $\mathrm{AD}$ compared to AMCI individuals $(p=0.012)$.

Distinct transcription factors (TF) are activated during the differentiation of $\mathrm{T}$ cells into functional subsets. We analyzed T-Bet, ROR-C/ $\gamma$ and GATA-3 TF in all the individuals enrolled in the study. Results show an increase of $A \beta_{42}$-stimulated$\mathrm{CD} 8+\mathrm{T}$ cells expressing $\mathrm{T}$-bet in $\mathrm{AD}$ compared to aMCI $(p=<0.001)$. As T-bet is the TF expressed by cells that have differentiated into TH1 lymphocytes, and because such lymphocytes are characterized by the production of IFN $\gamma$, these results explain the increased production of this cytokine seen in $\mathrm{AD}$ (Table 3).

Table 2 | Results of DTI-based tractography.

\begin{tabular}{|c|c|c|c|c|c|c|}
\hline \multirow[b]{2}{*}{$\begin{array}{l}\text { CC } \\
\text { portion }\end{array}$} & \multicolumn{2}{|c|}{ aAMCI } & \multicolumn{2}{|c|}{$A D$} & \multicolumn{2}{|c|}{$\begin{array}{l}\text { Comparison } \\
\text { aMCl-AD (*) }\end{array}$} \\
\hline & $\begin{array}{l}\text { FA } \\
\text { Mean } \\
\text { (SD) }\end{array}$ & $\begin{array}{l}\text { MD } \\
\text { Mean } \\
\text { (SD) }\end{array}$ & $\begin{array}{l}\text { FA } \\
\text { Mean } \\
\text { (SD) }\end{array}$ & $\begin{array}{l}\text { MD } \\
\text { Mean } \\
\text { (SD) }\end{array}$ & $\begin{array}{l}\text { FA } \\
p \\
\text { value }\end{array}$ & $\begin{array}{l}\text { MD } \\
p \\
\text { value }\end{array}$ \\
\hline $\mathrm{CC} 1$ & $0.47(0.06)$ & $0.86(0.11)$ & $0.45(0.06)$ & $0.94(0.10)$ & n.s. & 0.013 \\
\hline $\mathrm{CC} 2$ & $0.59(0.06)$ & $0.90(0.09)$ & $0.54(0.07)$ & $0.98(0.11)$ & 0.009 & 0.012 \\
\hline $\mathrm{CC} 3$ & $0.52(0.06)$ & $0.97(0.07)$ & $0.49(0.05)$ & $1.04(0.09)$ & 0.021 & 0.018 \\
\hline CC4 & $0.50(0.07)$ & $1.04(0.11)$ & $0.48(0.07)$ & $1.12(0.13)$ & n.s. & 0.034 \\
\hline CC5 & $0.58(0.08)$ & $0.93(0.09)$ & $0.54(0.08)$ & $1.03(0.15)$ & n.s. & 0.009 \\
\hline CC6 & $0.59(0.09)$ & $1.01(0.20)$ & $0.57(0.09)$ & $1.13(0.24)$ & n.s. & 0.039 \\
\hline $\mathrm{CC} 7$ & $0.54(0.10)$ & $0.97(0.15)$ & $0.53(0.10)$ & $1.03(0.13)$ & n.s. & n.s. \\
\hline
\end{tabular}

Comparison between DTI-metrics (mean FA/MD) computed in the seven CC portions of the two groups (aMCl and $A D$ patients).

$A D$, Alzheimer's disease; aMCl, amnestic mild cognitive impairment; CC, corpus callosum; FA, fractional anisotropy; $M D$, mean diffusivity. $\left(^{*}\right) p$-values refer to t-test, significance level $p<0.05$
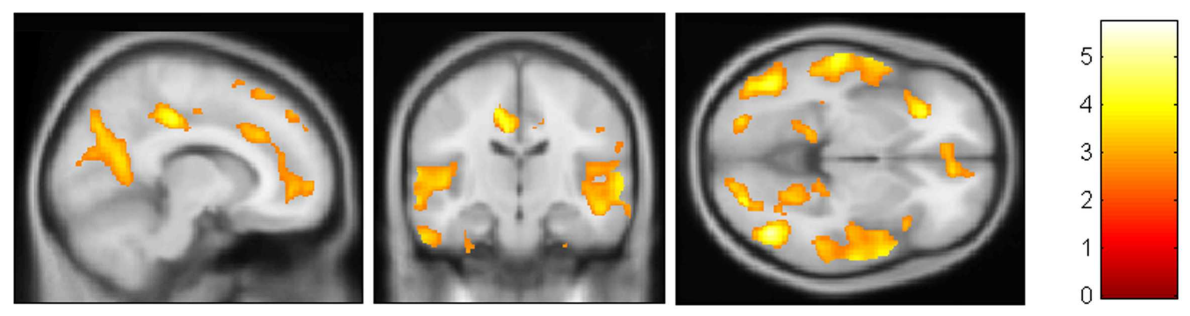

FIGURE 1 | VBM results. AD patients were found to be significantly more atrophic than $\mathrm{aMCl}$ in medial, anterior, and postero-inferior regions of temporal lobes (left $>$ right) and precuneus/posterior cingulate. (FDR $<0.05$ ) 


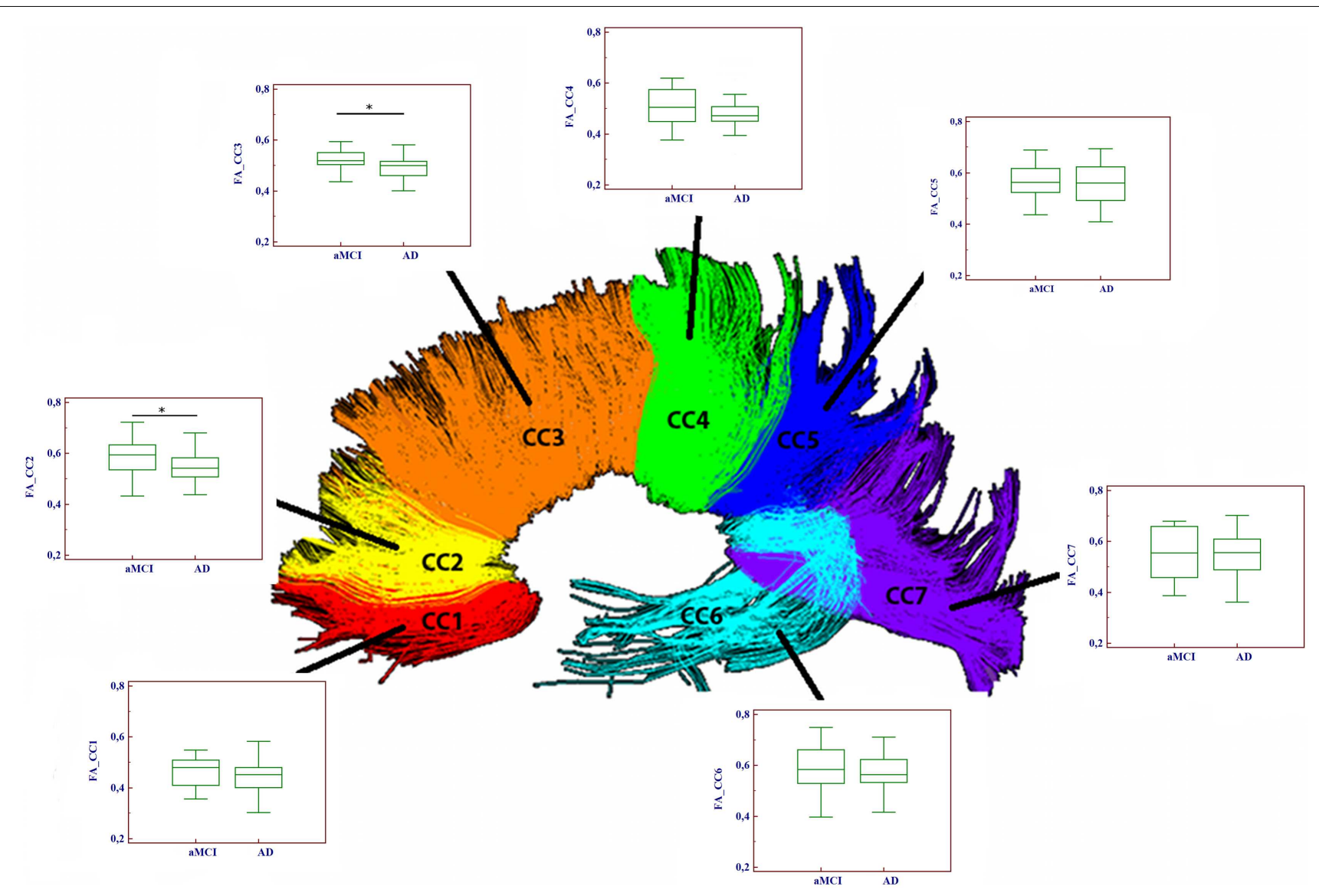

FIGURE 2 | Comparison of fractional anisotropy (FA) values between aMCI and AD patients in the seven portions of the corpus callosum (CC) Significant differences between the two groups were found only in two frontal CC portions (CC2 and CC3). $\left(^{*} p<0.05\right.$ ).

\section{Cytokine-producing CD14+ cells in A $\beta_{42}$-peptide-stimulated cell cultures}

The immune response is mediated by multiple cell types. To better approach the in vivo situation we examined IL-6-, IL-10-, IL-12p35-, IL-23-, and TGF $\beta$-expressing CD14+ monocytes in peripheral mononuclear cell cultures that had been stimulated with the non-immunogenic or with the $A \beta_{42}$-peptide. Results indicated that $A \beta_{42}$-stimulated TGF- $\beta$-producing CD14+ cells were significantly decreased in $\mathrm{AD}$ compared to aMCI $(p=0.003)$ (Table 3).

\section{PD-1-expressing CD4+ and CD8+ T lymphocytes and PD-L1-expressing CD14+ and CD19+ cells in $A \beta_{42}$-peptide-stimulated PBMC}

PD-1 on T lymphocytes binds PD-L1 on the surface of antigen presenting cells (APC) resulting in the dampening of adaptive immune-response via tolerization or apoptosis of antigenspecific T lymphocytes. $A \beta_{42}$-stimulated lymphocytes and monocytes (APC) were analyzed in PBMC isolated from the peripheral blood of patients. Results showed that both PD-1- and PD-L1expressing cells were reduced in $\mathrm{AD}$ compared to aMCI individuals, even if the differences did not reach statistical significance (Table 3). Again, no differences were observed when cells were incubated with the non-immunogenic peptides (data not shown).

\section{CORRELATIONS BETWEEN MRI AND IMMUNOLOGIC PARAMETERS}

Correlations were sought between the FA values that were significantly different between groups (CC2-CC3) and immunological parameters; separate analyses were performed for each group. Results showed significantly positive correlations in aMCI between the FA values for both $\mathrm{CC} 2$ and $\mathrm{CC} 3$ and circulating $\mathrm{A} \beta_{42}$ stimulated $\mathrm{CD} 4+/ \mathrm{BDNF}+, \mathrm{CD} 8+/ \mathrm{BDNF}+$, and $\mathrm{CD} 14+/ \mathrm{PD}-$ $\mathrm{L} 1+$ cells (Table 4). These correlations were not maintained in AD patients in whom, instead, an important positive correlation was detected between the CC2 FA and $\mathrm{A}_{42}$-stimulated CD4+/IL-21+ (inflammatory) lymphocytes $\left(R_{\mathrm{Sp}}=-0.41\right)$.

\section{DISCUSSION}

We investigated possible interactions among peripheral immunological parameters and brain disconnection in $\mathrm{AD}$ and aMCI individuals using DTI-based tractography. Analyses on brain disconnection focused on the CC, which appears as an ideal candidate for the investigation of WM damage in the different stages of the $\mathrm{AD}$ disease, due to its particular susceptibility to atrophy (Thomann et al., 2006; Chaim et al., 2007). This structure was 
Table 3 | CD4+, CD8+, and CD14+ cells that produce cytokines and CD4+, CD8+, CD14+, and CD19+ cells that express PD-1 or PD-L1 in patients with a diagnosis of either $A D$ or aMCI.

\begin{tabular}{|c|c|c|c|}
\hline & aMCI & AD & $p$-value $(*)$ \\
\hline $\mathrm{CD}^{+}{ }^{+} \mathrm{GATA}^{-}{ }^{+}$ & $0.40(0.00-1.25)$ & $0.05(0.00-0.70)$ & n.s. \\
\hline $\mathrm{CD}^{+}{ }^{+} \mathrm{ROR}-\mathrm{C}-\gamma^{+}$ & $0.16(0.07-0.40)$ & $0.10(0.00-0.38)$ & n.s. \\
\hline $\mathrm{CD} 4^{+} \mathrm{IFN} \gamma^{+}$ & $0.10(0.01-0.32)$ & $0.15(0.00-0.40)$ & n.s. \\
\hline $\mathrm{CD} 4^{+} \mid \mathrm{L}-17^{+}$ & $0.05(0.00-0.08)$ & $0.07(0.00-0.29)$ & n.s. \\
\hline $\mathrm{CD}_{4}^{+} \mathrm{IL}^{-9+}$ & $0.12(0.04-0.40)$ & $0.10(0.02-0.22)$ & n.s. \\
\hline $\mathrm{CD} 4^{+} \mathrm{IL}-21^{+}$ & $0.01(0.00-0.73)$ & $0.30(0.02-0.62)$ & n.s. \\
\hline $\mathrm{CD} 4^{+} \mathrm{IL}^{-22^{+}}$ & $0.20(0.10-0.50)$ & $0.42(0.10-0.70)$ & n.s. \\
\hline $\mathrm{CD}^{+}{ }^{+} \mathrm{BDNF}^{+}$ & $0.39(0.27-0.58)$ & $0.12(0.03-0.29)$ & 0.003 \\
\hline $\mathrm{CD}^{+}{ }^{+} \mathrm{BDNF}^{+}$ & $0.45(0.18-1.71)$ & $0.31(0.13-0.55)$ & n.s. \\
\hline $\mathrm{CD}^{+}$T-bet $^{+}$ & $0.01(0.00-0.05)$ & $1.22(0.09-3.16)$ & $<0.001$ \\
\hline $\mathrm{CD} 8^{+} \mathrm{IFN} \gamma^{+}$ & $0.02(0.00-0.065)$ & $0.065(0.01-0.15)$ & 0.0127 \\
\hline CD14+TGF- $\beta^{+}$ & $5.50(2.27-16.05)$ & $1.87(0.53-3.05)$ & 0.003 \\
\hline $\mathrm{CD} 14^{+} \mathrm{IL}_{-6}{ }^{+}$ & $15.50(1.27-38.77)$ & $10.11(2.20-16.00)$ & n.s. \\
\hline $\mathrm{CD} 14^{+} \mid \mathrm{L}-10^{+}$ & $3.95(2.43-13.88)$ & $2.05(0.53-12.82)$ & n.s. \\
\hline $\mathrm{CD}_{14}{ }^{+} \mathrm{IL}-12 \mathrm{p} 35^{+}$ & $0.6(0.00-1.82)$ & $0.18(0.02-1.4)$ & n.s. \\
\hline $\mathrm{CD} 14^{+} \mathrm{IL}^{-23^{+}}$ & $2.5(0.92-4.49)$ & $1.4(0.47-2.56)$ & n.s. \\
\hline $\mathrm{CD} 4^{+}{ }^{\mathrm{PD}}-1^{+}$ & $0.38(0.00-2.67)$ & $0.31(0.1-2.10)$ & n.s. \\
\hline $\mathrm{CD}^{+}{ }^{\mathrm{PD}}-1^{+}$ & $0.06(0.00-0.09)$ & $0.04(0.02-0.15)$ & n.s. \\
\hline CD19+PD-L1+ & $0.05(0.00-0.07)$ & $0.02(0.00-0.08)$ & n.s. \\
\hline CD14+PD-L1+ & $1.20(0.30-3.34)$ & $0.55(0.28-1.10)$ & n.s. \\
\hline
\end{tabular}

Percentages of $A \beta_{42}$-stimulated cells are shown; median, interquantile ranges, and statistical significance are indicated.

$A D$, Alzheimer's disease; $a \mathrm{MCl}$, amnestic mild cognitive impairment; IL, interleukin; BDNF, brain-derived neurotrophic factor; ROR, retinoid acid-related orphan receptor; T-bet, T-box expressed in T cells; IFN- $\gamma$, interferon-gamma; TGF- $\beta$, transforming growth factor-beta; $P D-1$, programed death-1; PD-L1, programed death-1 ligand.

(*) $p$-Values refer to Mann-Whitney-U, significance level $p<0.05$.

In all cases, background results (i.e., \% of cytokines secreting or $P D$-expressing cells in PBMC incubated with non-immunogenic peptides) were subtracted from the values shown.

evaluated in its seven different parts, allowing the definition of a valued topographic distinction of WM damage in different stages of $\mathrm{AD}$ pathology.

MRI results showed that different WM damage pattern are seen in aMCI and AD. Thus DTI analysis on FA indicated that the frontal CC subregions are preserved in the prodromal stage of the pathology (higher FA values on FA CC2-CC3 in aMCI), and MD values were still preserved in aMCI in almost the whole CC (higher MD values in AD only in CC7). This appears interesting when considering the different type of information provided by the two DTI indices: MD is more fit at analyzing extracellular volume alterations whereas FA better investigates impairments in the axonal integrity (Werring et al., 1999; Rovaris et al., 2005), independently of the gross tissue loss (Burzynska et al., 2010). When these indexes are analyzed together, lower FA and higher MD values suggests a loss of cortical regions related to the phenomenon of the anterograde Wallerian degeneration (Xie et al., 2006; Damoiseaux et al., 2009) whereas areas characterized by lower FA without particular changes on MD indicate the presence
Table 4 | Correlations between MRI (DTI-metrics) and immunological parameters.

\begin{tabular}{llll}
\hline Variable & With variable & $\boldsymbol{R}_{\text {Sp }}(\mathbf{9 5 \%} \mathbf{C . L .})$ & $\boldsymbol{P}$ value \\
\hline aMCl & & & \\
FA_CC2 & CD14+/PD-L1+ & $0.53(0.01 ; 0.82)$ & 0.0389 \\
FA_CC3 & CD14+/PD-L1+ & $0.66(0.21 ; 0.87)$ & 0.0057 \\
FA_CC2 & CD4+/BDNF+ & $0.64(0.08 ; 0.88)$ & 0.0226 \\
FA_CC3 & CD4+/BDNF+ & $0.62(0.05 ; 0.88)$ & 0.0284 \\
FA_CC2 & CD8+/BDNF+ & $0.69(0.16 ; 0.90)$ & 0.0116 \\
FA_CC3 & CD8+/BDNF+ & $0.81(0.40 ; 0.94)$ & 0.0008 \\
AD & & & \\
FA_CC3 & CD4+/IL-21+ & $-0.41(-0.68 ;-0.02)$ & 0.0346
\end{tabular}

$A D$, Alzheimer's disease; $a \mathrm{MCl}$, amnestic mild cognitive impairment; $C C$, corpus callosum; $F A$, fractional anisotropy; $R_{S_{0}}$, Spearman correlation coefficient and $95 \%$ confidence limits performed by Fisher's $Z$ transformation.

of a possible myelin breakdown process related to amyloid and tau deposits (Bartzokis, 2011; Lu et al., 2013). Results herein indicate that in aMCI brain disconnection mechanism are mainly due to myelin breakdown (lower FA without increase in MD). Interestingly, this process is located in almost the whole CC with the exclusion of frontal and parietal CC regions. This finding appears in line with previous results (Parente et al., 2008; Di Paola et al., 2010; Agosta et al., 2011; Preti et al., 2012), describing AD as part of a continuum of clinical and biological phenomena with a posterior to anterior pathophysiological evolution. This early damage of the posterior CC subregions in $\mathrm{AD}$ reflects the accumulation of proteinaceous deposits, such as $\mathrm{A} \beta$ and tau, involving initially the medial-temporal regions (Brun and Englund, 2002). These results are consistent with MRI-GM structural data and with the wellknown pattern of distribution of neuropathological changes typical of AD neurodegeneration, which starts from medial-temporal regions then spreads to medial-parietal cortex and orbitofrontal regions and finally to other neocortical association areas (Busatto et al., 2008).

Results of immunological analyses performed in the same individuals showed that, upon $A \beta_{42}$-stimulation, BDNF-producing $\mathrm{CD} 4+$ and TGF- $\beta$-producing CD14+ were significantly reduced, whereas IFN $\gamma$-producing and T-bet expressing CD8 $+\mathrm{T}$ cell were increased in $\mathrm{AD}$ compared to aMCI. These immune alterations were detected only in vitro $A \beta_{42}$-stimulated cell cultures suggesting that they are specific for the aMCI-AD spectrum. TGF- $\beta$ and BDNF are cytokines endowed with anti-inflammatory and neuroprotective function, even though recently these roles have become ambiguous. In particular TGF- $\beta$ is a pleiotropic cytokine characterized by mutually exclusive regulatory and inflammatory activities that are cellular and environmental-context dependent. When the inflammatory properties prevail, TGF- $\beta$ induces the differentiation of TH17 cells (Sanjabi et al., 2009). The observation that in aMCI TH17-produced cytokines (IL-17, IL-21, IL-22) were not detected seems to indicate that the biological significance of the increased production of TGF- $\beta$ seen in these patients is anti-inflammatory. In $\mathrm{AD}$ the recruitment of peripheral monocytes/macrophages within the CNS could contrast the formation 
and/or reduce the extension of $\beta$-amyloid plaques via multiple mechanisms mediated by innate and acquired immunity (Tan et al., 1999a,b, 2002a; Town et al., 2001, 2005a,b, 2008; Townsend et al., 2005; Hawkes and McLaurin, 2009). Thus, it has previously been suggested that innate immune responses, possibly triggered by TLR activation, play a role in the AD-associated neuroinflammation (Fassbender et al., 2004; Fiala et al., 2007; Walter et al., 2007; Malm et al., 2010; Liu et al., 2012; Saresella et al., 2014). Notably, in $\mathrm{AD}$ patients $\mathrm{T}$ cell in peripheral blood and infiltrating the brain are indeed activated and display memory phenotype (Shalit et al., 1995; Lombardi et al., 1999, 2004; Tan et al., 2002b; Togo et al., 2002); levels of IL-6 in plasma (Singh and Guthikonda, 1997), augmented production of IFN- $\gamma$ and TNF- $\alpha$ by natural killer (NK) cells (Solerte et al., 2000), and an increase in IL-1 $\beta$ associated with a concomitant decrease in IL-10 have been observed in AD (Arosio et al., 2004).

Activated T lymphocytes can nevertheless also mediate neuroprotection and promote neurogenesis by secreting BDNF. BDNF production was increased in aMCI; these results are is in line with data showing higher BDNF serum levels in preclinical stages of Alzheimer's disease (Laske et al., 2006; Angelucci et al., 2010) and with post-mortem examinations of $\mathrm{AD}$ brains indicating a significant increase of BDNF concentration in hippocampus and parietal cortex (Durany et al., 2000) and of its receptor TrkB in astrocytes and senile plaques (Connor et al., 1997). BDNF was recently suggested to also mediate neurodegeneration secondarily to its ability to induce nitric oxide production by astrocytes (Colombo et al., 2012). Nevertheless, the observation that in aMCI a correlation was detected between BDNF and preserved brain morphology seems to confirm a protective role for this cytokine. Notably, the correlations between MRI parameters and BDNF secreting immune cells were lost in AD in whom, instead, brain atrophy was positively correlated with IL-21-producing CD4+ T cells. This seems biologically important considering that IL-21 is a potent proinflammatory cytokine that plays a role in autoimmunity, including multiple sclerosis, and whose biological properties include the proliferation of $\mathrm{T}$ cells proliferation, the stimulation of NK activity, and the functional dampening of T reg cells (Leonard and Spolski, 2005; Spolski and Leonard, 2008; Raveney et al., 2013). Confirming the presence of inflammation is $\mathrm{AD}$ are also the results indicating that $\mathrm{TH} 1$-driven IFN $\gamma$ production by T-bet-expressing $(\mathrm{TH} 1)$ $\mathrm{CD} 8+\mathrm{T}$ lymphocytes was detected in these patients.

The preservation of brain morphology seen in aMCI was positively correlated with PD-L1 expressing CD14+ cells as well. PDL1 binds PD-1 and this interaction dampens immune responses, harnessing inflammation. Recent results showed that the expression of these molecules is significantly reduced in AD and aMCI, thus, in these diseases, fewer PD-1-expressing CD4+ T cells bind reduced quantities of $\mathrm{PD}-\mathrm{L} 1$ molecules on the surface of CD14+ APC. The interaction between PD-1 and PD-L1 regulates the reciprocal balance between $\mathrm{T}$ cell tolerance and activation, and recent data show that when PD-1 is engaged, the earliest events in T cell activation are blocked (Parry et al., 2005), indicating that PD-1 is a highly effective negative regulator of T-cell activation even when modestly expressed on T cell surface (Riley, 2009). Ligation of PD-L1 limits effectors' cells responses and prevents the triggering of immune-mediated tissue damage (Selenko-Gebauer et al., 2003; Keir et al., 2007; Sharpe et al., 2007). This antigenspecific phenomenon is achieved via the generation of IL-10, a powerful inflammation-dampening cytokine, and/or by the limitation of the proliferation of antigen-specific cells secondarily to the apoptosis of such cells (Dong et al., 2002; Dong and Chen, 2003; Trabattoni et al., 2003). IL-10 was comparable in AD and aMCI, suggesting that modulation of apoptosis could be mechanism associated with the preservation of brain tissues seen in aMCI. The positive correlations seen in these individuals between CC2 and CC3 and PD-L1 expressing immune cells could speculatively be justified as follows: more $\mathrm{A} \beta$ specific cells would undergo apoptosis secondarily to more frequent $\mathrm{PD}-1 / \mathrm{PD}-\mathrm{L} 1$ interactions; this would results in a reduced degree of neuroinflammation and, as a consequence, a better preservation of cortical architectures. This mechanism is lost in $\mathrm{AD}$, leading to impairments of such structures.

These immunological results stem from analyses performed on circulating immune cells, in the attempt to define immunologic diagnostic and prognostic correlates of disease that would be easily monitored and reproduced. The fact that we used peripheral blood lymphocytes instead than cells circulating in the cerebrospinal fluid (CSF) could apparently weaken our results. Because of ethical constrains, CSF examination could be performed in our patients only when necessary to exclude reversible causes of dementia. Nevertheless, the observations that immune cells continuously re-circulate throughout the body and migrate across the blood-brain barrier (BBB), and that such barrier is permeable to cytokines, lend support to our results.

In conclusion, the results herein seem to support the hypothesis that a disconnection syndrome in aMCI and AD could correlates with WM myelin breakdown caused by a neuroinflammation as pathophysiological substrate. The combination of DTI and neuroinflammation parameters might define possible surrogate biomarkers that could be useful in longitudinal studies and in clinical and pharmacological trials.

\section{REFERENCES}

Agosta, F., Pievani, M., Sala, S., Geroldi, C., Galluzzi, S., Frisoni, G. B., et al. (2011). White matter damage in Alzheimer disease and its relationship to gray matter atrophy. Radiology 258, 853-863. doi:10.1148/radiol.10101284

Angelucci, F., Spalletta, G., di Iulio, F., Ciaramella, A., Salani, F., Colantoni, L., et al. (2010). Alzheimer's disease (AD) and mild cognitive impairment (MCI) patients are characterized by increased BDNF serum levels. Curr. Alzheimer Res. 7, 15-20. doi:10.2174/156720510790274473

Arosio, B., Trabattoni, D., Galimberti, L., Bucciarelli, P., Fasano, F., Calabresi, C., et al. (2004). Interleukin-10 and interleukin-6 gene polymorphisms as risk factors for Alzheimer's disease. Neurobiol. Aging 25, 1009-1015. doi:10.1016/j. neurobiolaging.2003.10.009

Ashburner, J., and Friston, K. J. (2005). Unified segmentation. Neuroimage 26, 839-851. doi:10.1016/j.neuroimage.2005.02.018

Bartzokis, G. (2011). Alzheimer's disease as homeostatic responses to agerelated myelin breakdown. Neurobiol. Aging 32, 1341-1371. doi:10.1016/j. neurobiolaging.2009.08.007

Bauer, J., Strauss, S., Schreiter-Gasser, U., Ganter, U., Schlegel, P., Witt, I., et al. (1991). Interleukin-6 and alpha-2-macroglobulin indicate an acute-phase state in Alzheimer's disease cortices. FEBS Lett. 285, 111-114. doi:10.1016/00145793(91)80737-N

Bermejo, P., Martin-Aragon, S., Benedi, J., Susin, C., Felici, E., Gil, P., et al. (2008). Differences of peripheral inflammatory markers between mild cognitive impairment and Alzheimer's disease. Immunol. Lett. 117, 198-202. doi:10.1016/j.imlet. 2008.02.002 
Bozzali, M., Falini, A., Franceschi, M., Cercignani, M., Zuffi, M., Scotti, G., et al. (2002). White matter damage in Alzheimer's disease assessed in vivo using diffusion tensor magnetic resonance imaging. J. Neurol. Neurosurg. Psychiatr. 72, 742-746. doi:10.1136/jnnp.72.6.742

Bozzali, M., Padovani, A., Caltagirone, C., and Borroni, B. (2011). Regional grey matter loss and brain disconnection across Alzheimer disease evolution. Curr. Med. Chem. 18, 2452-2458. doi:10.2174/092986711795843263

Brun, A., and Englund, E. (2002). Regional pattern of degeneration in Alzheimer's disease: neuronal loss and histopathological grading. Histopathology 5, 549-564.

Burzynska, A. Z., Preuschhof, C., Backman, L., Nyberg, L., Li, S. C., Lindenberger, U., et al. (2010). Age-related differences in white matter microstructure: region-specific patterns of diffusivity. Neuroimage 49, 2104-2112. doi:10.1016/ j.neuroimage.2009.09.041

Busatto, G. F., Diniz, B. S., and Zanetti, M. V. (2008). Voxel-based morphometry in Alzheimer's disease. Exp. Rev. Neurother 8, 1691-1702. doi:10.1586/14737175.8. 11.1691

Chaim, T. M., Duran, F. L., Uchida, R. R., Perico, C. A., de Castro, C. C., and Busatto, G. F. (2007). Volumetric reduction of the corpus callosum in Alzheimer's disease in vivo as assessed with voxel-based morphometry. Psychiatry Res. 154, 59-68. doi:10.1016/j.pscychresns.2006.04.003

Colombo, E., Cordiglieri, C., Melli, G., Newcombe, J., Krumbholz, M., Parada, L. F., et al. (2012). Stimulation of the neurotrophin receptor TrkB on astrocytes drives nitric oxide production and neurodegeneration. J. Exp. Med. 209, 521-535. doi:10.1084/jem.20110698

Connor, B., Young, D., Yan, Q., Faull, R. L., Synek, B., and Dragunow, M. (1997). Brain-derived neurotrophic factor is reduced in Alzheimer's disease. Brain Res. Mol. Brain Res. 49, 71-81. doi:10.1016/S0169-328X(97)00125-3

Damoiseaux, J. S., Smith, S. M., Witter, M. P., Sanz-Arigita, E. J., Barkhof, F., Scheltens, P., et al. (2009). White matter tract integrity in aging and Alzheimer's disease. Hum. Brain Mapp. 30, 1051-1059. doi:10.1002/hbm.20563

Di Paola, M., Di Iulio, F., Cherubini, A., Blundo, C., Casini, A. R., Sancesario, G., et al. (2010). When, where, and how the corpus callosum changes in MCI and AD: a multimodal MRI study. Neurology 74, 1136-1142. doi:10.1212/WNL. $0 \mathrm{~b} 013 \mathrm{e} 3181 \mathrm{~d} 7 \mathrm{~d} 8 \mathrm{cb}$

Dong, H., and Chen, L. (2003). B7-H1 pathway and its role in the evasion of tumor immunity. J. Mol. Med. (Berl.) 81, 281-287. doi:10.1007/s00109-003-0430-2

Dong, J., Mrabet, O., Moze, E., Li, K., and Neveu, P. J. (2002). Lateralization and catecholaminergic neuroimmunomodulation: prazosin, an alpha1/alpha2-adrenergic receptor antagonist, suppresses interleukin-1 and increases interleukin-10 production induced by lipopolysaccharides. Neuroimmunomodulation 10, 163-168. doi:10.1159/000067178

Drzezga, A., Becker, J. A., Van Dijk, K. R., Sreenivasan, A., Talukdar, T., Sullivan, C., et al. (2011). Neuronal dysfunction and disconnection of cortical hubs in non-demented subjects with elevated amyloid burden. Brain 134, 1635-1646. doi:10.1093/brain/awr066

Duan, J. H., Wang, H. Q., Xu, J., Lin, X., Chen, S. Q., Kang, Z., et al. (2006). White matter damage of patients with Alzheimer's disease correlated with the decreased cognitive function. Surg. Radiol. Anat. 28, 150-156. doi:10.1007/s00276-0060111-2

Durany, N., Michel, T., Kurt, J., Cruz-Sanchez, F. F., Cervas-Navarro, J., and Riederer, P. (2000). Brain-derived neurotrophic factor and neurotrophin-3 levels in Alzheimer's disease brains. Int. J. Dev. Neurosci. 18, 807-813. doi:10.1016/S07365748(00)00046-0

Fassbender, K., Walter, S., Kühl, S., Landmann, R., Ishii, K., Bertsch, T., et al. (2004). The LPS receptor (CD14) links innate immunity with Alzheimer's disease. FASEB J. 1, 203-205. doi:10.1096/fj.03-0364fje

Fiala, M., Liu, P. T., Espinosa-Jeffrey, A., Rosenthal, M. J., Bernard, G., Ringman, J. M., et al. (2007). Innate immunity and transcription of MGAT-III and tolllike receptors in Alzheimer's disease patients are improved by bisdemethoxycurcumin. Proc. Natl. Acad. Sci. U.S.A. 104, 12849-12854. doi:10.1073/pnas. 0701267104

Francisco, L. M., Sage, P. T., and Sharpe, A. H. (2010). The PD-1 pathway in tolerance and autoimmunity. Immunol. Rev. 236, 219-242. doi:10.1111/j.1600-065X.2010. 00923.x

Gili, T., Cercignani, M., Serra, L., Perri, R., Giove, F., Maraviglia, B., et al. (2011). Regional brain atrophy and functional disconnection across Alzheimer's disease evolution. J. Neurol. Neurosurg. Psychiatry 82, 58-66. doi:10.1136/jnnp.2009. 199935
Grundman, M., Petersen, R. C., Ferris, S. H., Thomas, R. G., Aisen, P. S., Bennett, D. A., et al. (2004). Mild cognitive impairment can be distinguished from Alzheimer disease and normal aging for clinical trials. Arch. Neurol. 61, 59-66. doi:10.1001/archneur.61.1.59

Hamilton, M. (1960). A rating scale for depression. J. Neurol. Neurosurg. Psychiatry 23, 56-62. doi:10.1136/jnnp.23.1.56

Hawkes, C. A., and McLaurin, J. (2009). Selective targeting of perivascular macrophages for clearance of beta-amyloid in cerebral amyloid angiopathy. Proc. Natl. Acad. Sci. U.S.A. 106, 1261-1266. doi:10.1073/pnas.0805453106

Kakeda, S., and Korogi, Y. (2010). The efficacy of a voxel-based morphometry on the analysis of imaging in schizophrenia, temporal lobe epilepsy, and Alzheimer's disease/mild cognitive impairment: a review. Neuroradiology 52, 711-721. doi:10.1007/s00234-010-0717-2

Karas, G., Sluimer, J., Goekoop, R., van der Flier, W., Rombouts, S. A., Vrenken, H., et al. (2008). Amnestic mild cognitive impairment: structural MR imaging findings predictive of conversion to Alzheimer disease. AJNR Am. J. Neuroradiol. 29, 944-949. doi:10.3174/ajnr.A0949

Ke, Z. J., Bowen, W. M., and Gibson, G. E. (2006). Peripheral inflammatory mechanisms modulate microglial activation in response to mild impairment of oxidative metabolism. Neurochem. Int. 49, 548-556. doi:10.1016/j.neuint.2006.04.011

Keir, M. E., Francisco, L. M., and Sharpe, A. H. (2007). PD-1 and its ligands in T-cell immunity. Curr. Opin. Immunol. 19, 309-314. doi:10.1016/j.coi.2007.04.012

Laske, C., Stransky, E., Leyhe, T., Eschweiler, G. W., Wittorf, A., Richartz, E., et al. (2006). Stage-dependent BDNF serum concentrations in Alzheimer's disease. J. Neural Transm. 113, 1217-1224. doi:10.1007/s00702-005-0397-y

Le Bihan, D. (2003). Looking into the functional architecture of the brain with diffusion MRI. Nat. Rev. Neurosci. 4, 469-480. doi:10.1038/nrn1119

Lebel, C., Caverhill-Godkewitsch, S., and Beaulieu, C. (2010). Age-related regional variations of the corpus callosum identified by diffusion tensor tractography. Neuroimage 52, 20-31. doi:10.1016/j.neuroimage.2010.03.072

Leonard, W. J., and Spolski, R. (2005). Interleukin-21: a modulator of lymphoid proliferation, apoptosis and differentiation. Nat. Rev. Immunol. 5, 688-698. doi:10.1038/nri1688

Leube, D. T., Weis, S., Freymann, K., Erb, M., Jessen, F., Heun, R., et al. (2008). Neural correlates of verbal episodic memory in patients with MCI and Alzheimer's disease - a VBM study. Int. J. Geriatr. Psychiatry 23, 1114-1118. doi:10.1002/gps. 2036

Liu, S., Liu, Y., Hao, W., Wolf, L., Kiliaan, A. J., Penke, B., et al. (2012). TLR2 is a primary receptor for Alzheimer's amyloid $\beta$ peptide to trigger neuroinflammatory activation. J. Immunol. 3, 1098-1107. doi:10.4049/jimmunol.1101121

Lombardi, V. R., Fernández-Novoa, L., Etcheverria, I., Seoane, S., and Cacabelos, R. (2004). Association between APOE epsilon4 allele and increased expression of CD95 on T cells from patients with Alzheimer's disease. Methods Find. Exp. Clin. Pharmacol. 26, 523-529. doi:10.1358/mf.2004.26.7.863735

Lombardi, V. R., Garcia, M., Rey, L., and Cacabelos, R. (1999). Characterization of cytokine production, screening of lymphocyte subset patterns and in vitro apoptosis in healthy and Alzheimer's Disease (AD) individuals. J. Neuroimmunol. 97, 163-171. doi:10.1016/S0165-5728(99)00046-6

Lu, P. H., Lee, G. J., Tishler, T. A., Meghpara, M., Thompson, P. M., and Bartzokis, G. (2013). Myelin breakdown mediates age-related slowing in cognitive processing speed in healthy elderly men. Brain Cogn. 81, 131-138. doi:10.1016/j.bandc.2012.09.006

Magni, E., Binetti, G., Bianchetti, A., Rozzini, R., and Trabucchi, M. (1996). MiniMental State Examination: a normative study in Italian elderly population. Eur. J. Neurol. 3, 198-202. doi:10.1111/j.1468-1331.1996.tb00423.x

Malm, T., Koistinaho, M., Muona, A., Magga, J., and Koistinaho, J. (2010). The role and therapeutic potential of monocyte cells in Alzheimer's disease. Glia 8, 889-900. doi:10.1002/glia.20973

McKhann, G. M., Knopman, D. S., Chertkow, H., Hyman, B. T., Jack, C. R. Jr, Kawas, C. H., et al. (2011). The diagnosis of dementia due to Alzheimer's disease: recommendations from the National Institute on Aging-Alzheimer's Association workgroups on diagnostic guidelines for Alzheimer's disease. Alzheimers Dement. 7, 263-269. doi:10.1016/j.jalz.2011.03.005

Morris, J. C. (1993). The Clinical Dementia Rating (CDR): current version and scoring rules. Neurology 43, 2412-2414. doi:10.1212/WNL.43.11.2412-a

Naggara, O., Oppenheim, C., Rieu, D., Raoux, N., Rodrigo, S., Dalla Barba, G., et al. (2006). Diffusion tensor imaging in early Alzheimer's disease. Psychiatry Res. 146, 243-249. doi:10.1016/j.pscychresns.2006.01.005 
Parente, D. B., Gasparetto, E. L., da Cruz, L. C. Jr, Domingues, R. C., Baptista, A. C., Carvalho, A. C., et al. (2008). Potential role of diffusion tensor MRI in the differential diagnosis of mild cognitive impairment and Alzheimer's disease. AJR Am. J. Roentgenol. 190, 1369-1374. doi:10.2214/AJR.07.2617

Parry, R. V., Chemnitz, J. M., Frauwirth, K. A., Lanfranco, A. R., Braunstein, I., Kobayashi, S. V., et al. (2005). CTLA-4 and PD-1 receptors inhibit T-cell activation by distinct mechanisms. Mol. Cell. Biol. 25, 9543-9553. doi:10.1128/MCB. 25.21.9543-9553.2005

Patenaude, B., Smith, S. M., Kennedy, D. N., and Jenkinson, M. (2011). A Bayesian model of shape and appearance for subcortical brain segmentation. Neuroimage 56, 907-922. doi:10.1016/j.neuroimage.2011.02.046

Petersen, R. C. (2004). Mild cognitive impairment as a diagnostic entity. J. Intern. Med. 256, 183-194. doi:10.1111/j.1365-2796.2004.01388.x

Preti, M. G., Baglio, F., Lagana, M. M., Griffanti, L., Nemni, R., Clerici, M., et al. (2012). Assessing corpus callosum changes in Alzheimer's disease: comparison between tract-based spatial statistics and atlas-based tractography. PLOS ONE 7:e35856. doi:10.1371/journal.pone.0035856

Raveney, B. J., Oki, S., and Yamamura, T. (2013). Nuclear receptor NR4A2 orchestrates Th17 cell-mediated autoimmune inflammation via IL-21 signalling. PLoS ONE 8:e56595. doi:10.1371/journal.pone.0056595

Remarque, E. J., Bollen, E. L., Weverling-Rijnsburger, A. W., Laterveer, J. C., Blauw, G. J., and Westendorp, R. G. (2001). Patients with Alzheimer's disease display a pro-inflammatory phenotype. Exp. Gerontol. 36, 171-176. doi:10.1016/S05315565(00)00176-5

Riley, J. L. (2009). PD-1 signaling in primary T cells. Immunol. Rev. 229, 114-125. doi:10.1111/j.1600-065X.2009.00767.x

Risacher, S. L., Saykin, A. J., West, J. D., Shen, L., Firpi, H. A., McDonald, B. C., et al. (2009). Baseline MRI predictors of conversion from MCI to probable AD in the ADNI cohort. Curr. Alzheimer Res. 6, 347-361. doi:10.2174/ 156720509788929273

Rosen, W. G., Terry, R. D., Fuld, P. A., Katzman, R., and Peck, A. (1980). Pathological verification of ischemic score in differentiation of dementias. Ann. Neurol. 7, 486-488. doi:10.1002/ana.410070516

Rovaris, M., Gass, A., Bammer, R., Hickman, S. J., Ciccarelli, O., Miller, D. H., et al. (2005). Diffusion MRI in multiple sclerosis. Neurology 65, 1526-1532. doi:10.1212/01.wnl.0000184471.83948.e0

Sanjabi, S., Zenewicz, L. A., Kamanaka, M., and Flavell, R. A. (2009). Antiinflammatory and pro-inflammatory roles of TGF-beta, IL-10, and IL-22 in immunity and autoimmunity. Curr. Opin. Pharmacol. 9, 447-453. doi:10.1016/ j.coph.2009.04.008

Saresella, M., Calabrese, E., Marventano, I., Piancone, F., Gatti, A., Alberoni, M., et al. (2011). Increased activity of Th-17 and Th-9 lymphocytes and a skewing of the post-thymic differentiation pathway are seen in Alzheimer's disease. Brain Behav. Immun. 25, 539-547. doi:10.1016/j.bbi.2010.12.004

Saresella, M., Calabrese, E., Marventano, I., Piancone, F., Gatti, A., Calvo, M. G., et al. (2010). PD1 negative and PD1 positive CD4+ T regulatory cells in mild cognitive impairment and Alzheimer's disease. J. Alzheimers Dis. 21, 927-938. doi:10.3233/JAD-2010-091696

Saresella, M., Calabrese, E., Marventano, I., Piancone, F., Gatti, A., Farina, E., et al. (2012). A potential role for the PD1/PD-L1 pathway in the neuroinflammation of Alzheimer's disease. Neurobiol. Aging 33, 624.e11-22. doi:10.1016/j. neurobiolaging.2011.03.004

Saresella, M., Marventano, I., Calabrese, E., Piancone, F., Rainone, V., Gatti, A., et al. (2014). A complex proinflammatory role for peripheral monocytes in Alzheimer's disease. J. Alzheimers Dis. 38, 403-413. doi:10.3233/JAD- 131160

Saresella, M., Marventano, I., Guerini, F. R., Zanzottera, M., Delbue, S., Marchioni, E., et al. (2008). Myelin basic protein-specific T lymphocytes proliferation and programmed cell death in demyelinating diseases. Clin. Immunol. 129, 509-517. doi:10.1016/j.clim.2008.08.010

Selenko-Gebauer, N., Majdic, O., Szekeres, A., Hofler, G., Guthann, E., Korthauer, U., et al. (2003). B7-H1 (programmed death-1 ligand) on dendritic cells is involved in the induction and maintenance of T cell anergy. J. Immunol. 170, 3637-3644.

Serra, L., Cercignani, M., Lenzi, D., Perri, R., Fadda, L., Caltagirone, C., et al. (2010). Grey and white matter changes at different stages of Alzheimer's disease. J. Alzheimers Dis 19, 147-159. doi:10.3233/JAD-2010-1223

Shalit, F., Sredni, B., Brodie, C., Kott, E., and Huberman, M. (1995). T lymphocyte subpopulations and activation markers correlate with severity of Alzheimer's disease. Clin. Immunol. Immunopathol. 75, 246-250. doi:10.1006/clin.1995.1078
Sharpe, A. H., Wherry, E. J., Ahmed, R., and Freeman, G. J. (2007). The function of programmed cell death 1 and its ligands in regulating autoimmunity and infection. Nat. Immunol. 8, 239-245. doi:10.1038/ni1443

Singh, V. K., and Guthikonda, P. (1997). Circulating cytokines in Alzheimer's disease. J. Psychiatr. Res. 31, 657-660. doi:10.1016/S0022-3956(97)00023-X

Solerte, S. B., Cravello, L., Ferrari, E., and Fioravanti, M. (2000). Overproduction of IFN-gamma and TNF- $\alpha$ from natural killer (NK) cells is associated with abnormal NK reactivity and cognitive derangement in Alzheimer's disease. Ann. N. Y. Acad. Sci. 917, 331-340. doi:10.1111/j.1749-6632.2000.tb05399.x

Speciale, L., Calabrese, E., Saresella, M., Tinelli, C., Mariani, C., Sanvito, L., et al. (2007). Lymphocyte subset patterns and cytokine production in Alzheimer's disease patients. Neurobiol. Aging 28, 1163-1169. doi:10.1016/j.neurobiolaging. 2006.05.020

Sperling, R. A., Aisen, P. S., Beckett, L. A., Bennett, D. A., Craft, S., Fagan, A. M., et al. (2011). Toward defining the preclinical stages of Alzheimer's disease: recommendations from the National Institute on Aging-Alzheimer's Association workgroups on diagnostic guidelines for Alzheimer's disease. Alzheimers Dement. 7, 280-292. doi:10.1016/j.jalz.2011.03.003

Spolski, R., and Leonard, W. J. (2008). The Yin and Yang of interleukin-21 in allergy, autoimmunity and cancer. Curr. Opin. Immunol. 20, 295-301. doi:10.1016/j.coi. 2008.02.004

Strauss, S., Bauer, J., Ganter, U., Jonas, U., Berger, M., and Volk, B. (1992). Detection of interleukin-6 and alpha 2-macroglobulin immunoreactivity in cortex and hippocampus of Alzheimer's disease patients. Lab. Invest. 66, 223-230.

Takahashi, S., Yonezawa, H., Takahashi, J., Kudo, M., Inoue, T., and Tohgi, H. (2002). Selective reduction of diffusion anisotropy in white matter of Alzheimer disease brains measured by 3.0 Tesla magnetic resonance imaging. Neurosci. Lett. 332, 45-48. doi:10.1016/S0304-3940(02)00914-X

Tan, J., Town, T., Abdullah, L., Wu, Y., Placzek, A., Small, B., et al. (2002a). CD45 isoform alteration in CD4 $+\mathrm{T}$ cells as a potential diagnostic marker of Alzheimer's disease. J. Neuroimmunol. 132, 164-172. doi:10.1016/S0165-5728(02)00309-0

Tan, J., Town, T., Crawford, F., Mori, T., DelleDonne, A., Crescentini, R., et al. (2002b). Role of CD40 ligand in amyloidosis in transgenic Alzheimer's mice. Nat. Neurosci. 5, 1288-1293. doi:10.1038/nn968

Tan, J., Town, T., Paris, D., Mori, T., Suo, Z., Crawford, F., et al. (1999a). Microglial activation resulting from CD40-CD40L interaction after beta-amyloid stimulation. Science 286, 2352-2355. doi:10.1126/science.286.5448.2352

Tan, J., Town, T., Suo, Z., Wu, Y., Song, S., Kundtz, A., et al. (1999b). Induction of CD40 on human endothelial cells by Alzheimer's beta-amyloid peptides. Brain Res. Bull. 50, 143-148. doi:10.1016/S0361-9230(99)00122-7

Thomann, P. A., Wustenberg, T., Pantel, J., Essig, M., and Schroder, J. (2006). Structural changes of the corpus callosum in mild cognitive impairment and Alzheimer's disease. Dement. Geriatr. Cogn. Disord. 21, 215-220. doi:10.1159/ 000090971

Togo, T., Akiyama, H., Iseki, E., Kondo, H., Ikeda, K., Kato, M., et al. (2002). Occurrence of T cells in the brain of Alzheimer's disease and other neurological diseases. J. Neuroimmunol. 124, 83-92. doi:10.1016/S0165-5728(01)00496-9

Town, T., Laouar, Y., Pittenger, C., Mori, T., Szekely, C. A., Tan, J., et al. (2008) Blocking TGF-beta-Smad2/3 innate immune signaling mitigates Alzheimer-like pathology. Nat. Med. 14, 681-687. doi:10.1038/nm1781

Town, T., Nikolic, V., and Tan, J. (2005a). The microglial "activation" continuum: from innate to adaptive responses. J. Neuroinflammation 2, 24. doi:10.1186/1742 2094-2-24

Town, T., Tan, J., Flavell, R. A., and Mullan, M. (2005b). T-cells in Alzheimer's disease. Neuromolecular Med. 7, 255-264. doi:10.1385/NMM:7:3:255

Town, T., Tan, J., and Mullan, M. (2001). CD40 signaling and Alzheimer's disease pathogenesis. Neurochem. Int. 39, 371-380. doi:10.1016/S0197-0186(01) 00044-4

Townsend, K. P., Town, T., Mori, T., Lue, L. F., Shytle, D., Sanberg, P. R., et al. (2005). CD40 signaling regulates innate and adaptive activation of microglia in response to amyloid beta-peptide. Eur. J. Immunol. 35, 901-910. doi:10.1002/ eji.200425585

Trabattoni, D., Saresella, M., Biasin, M., Boasso, A., Piacentini, L., Ferrante, P., et al. (2003). B7-H1 is up-regulated in HIV infection and is a novel surrogate marker of disease progression. Blood 101, 2514-2520. doi:10.1182/blood-200210-3065

Vemuri, P., Weigand, S. D., Knopman, D. S., Kantarci, K., Boeve, B. F., Petersen, R. C., et al. (2011). Time-to-event voxel-based techniques to assess regional 
atrophy associated with MCI risk of progression to AD. Neuroimage 54, 985-991. doi:10.1016/j.neuroimage.2010.09.004

Walter, S., Letiembre, M., Liu, Y., Heine, H., Penke, B., Hao, W., et al. (2007). Role of the toll-like receptor 4 in neuroinflammation in Alzheimer's disease. Cell. Physiol. Biochem. 6, 947-956. doi:10.1159/000110455

Werring, D. J., Clark, C. A., Barker, G. J., Thompson, A. J., and Miller, D. H. (1999). Diffusion tensor imaging of lesions and normal-appearing white matter in multiple sclerosis. Neurology 52, 1626-1632. doi:10.1212/WNL.52.8.1626

Xie, S., Xiao, J. X., Gong, G. L., Zang, Y. F., Wang, Y. H., Wu, H. K., et al. (2006). Voxel-based detection of white matter abnormalities in mild Alzheimer disease. Neurology 66, 1845-1849. doi:10.1212/01.wnl.0000219625.77625.aa

Zhang, Y., Schuff, N., Jahng, G. H., Bayne, W., Mori, S., Schad, L., et al. (2007). Diffusion tensor imaging of cingulum fibers in mild cognitive impairment and Alzheimer disease. Neurology 68, 13-19. doi:10.1212/01.wnl.0000250326. 77323.01

Zipp, F., and Aktas, O. (2006). The brain as a target of inflammation: common pathways link inflammatory and neurodegenerative diseases. Trends Neurosci. 29, 518-527. doi:10.1016/j.tins.2006.07.006
Conflict of Interest Statement: The authors declare that the research was conducted in the absence of any commercial or financial relationships that could be construed as a potential conflict of interest.

Received: 30 September 2013; accepted: 11 November 2013; published online: 25 November 2013.

Citation: Baglio F, Saresella M, Preti MG, Cabinio M, Griffanti L, Marventano I, Piancone F, Calabrese E, Nemni $R$ and Clerici M (2013) Neuroinflammation and brain functional disconnection in Alzheimer's disease. Front. Aging Neurosci. 5:81. doi: 10.3389/fnagi.2013.00081

This article was submitted to the journal Frontiers in Aging Neuroscience.

Copyright (C) 2013 Baglio, Saresella, Preti, Cabinio, Griffanti, Marventano, Piancone, Calabrese, Nemni and Clerici. This is an open-access article distributed under the terms of the Creative Commons Attribution License (CC BY). The use, distribution or reproduction in other forums is permitted, provided the original author(s) or licensor are credited and that the original publication in this journal is cited, in accordance with accepted academic practice. No use, distribution or reproduction is permitted which does not comply with these terms. 